\title{
DECOMPOSIÇÃO TÉRMICA DA MADEIRA DE DUAS ESPÉCIES DA CAATINGA: Caesalpinia pyramidalis E Aspidosperma pyrifolium
}

\section{THERMAL DECOMPOSITION OF CAATINGA WOOD TWO SPECIES: Caesalpinia pyramidalis $\mathrm{E}$ Aspidosperma pyrifolium}

\author{
Cynthia Patricia de Sousa Santos ${ }^{1}$; Sarah Esther de Lima Costa ${ }^{2}$; Izabelle Rodrigues \\ Ferreira Gomes ${ }^{3}$; Rosimeire Cavalcante dos Santos ${ }^{4}$, Vinícius Resende de Castro ${ }^{5}$
}

DOI: https://doi.org/10.31692/978-65-991061-7-0.241-249

\section{RESUMO}

O processo de conversão da madeira em energia torna-se complexo a partir da inferência sobre a heterogeneidade desse material, que configura diferentes proporções da composição química da madeira entre as diversas espécies. De maneira a entender o comportamento da madeira em diferentes temperaturas, este trabalho teve como objetivo verificar a decomposição térmica da madeira de duas espécies florestais da Caatinga, a partir do método de termogravimetria (TGA), e avaliar o teor de cinzas, carbono fixo e voláteis, a fim de indicar a potencialidade das mesmas para fins energéticos. Foram coletados três indivíduos da espécie Caesalpinia pyramidalis e dois indivíduos da espécie Aspidosperma pyrifolium, localizados na Fazenda Milhã/Poço da Pedra, RN. De cada indivíduo foram retirados discos com $15 \mathrm{~cm}$ de espessura na faixa da altura do diâmetro do peito $(1,30 \mathrm{~m})$. Para realização da análise química imediata foi adotada a metodologia ASTM E872-1982 (ASTM, 2006) para matérias voláteis, ASTM E1755-2001 (ASTM, 2007) para o teor de cinzas e o carbono fixo foi estimado pela diferença desses dois teores. A análise termogravimétrica foi realizada utilizando uma faixa de temperatura de 25 a $750{ }^{\circ} \mathrm{C}$ sob atmosfera de $\mathrm{N}^{2}$. e razão de aquecimento de $10{ }^{\circ} \mathrm{C}$. $\min ^{-1}$ e foi adotado duas repetições para cada espécie "A e B". Na análise estatística foram utilizados os testes Kolmogorov-Smirnov, Levene, teste $\mathrm{F}$ da análise de variância e considerou-se à $95 \%$ de probabilidade. Para as amostras "A" foi verificada a perda de massa entre 300 a $450{ }^{\circ} \mathrm{C}$ de aproximadamente $53,65 \%$ para as amostras $\mathrm{C} 4 \mathrm{~A}$ (Caesalpinia pyramidalis) e P2A (Aspidosperma pyrifolium) e de $60 \%$ para as amostras C2A e C3A (Caesalpinia pyramidalis). Para as amostras "B" foi verificada a perda de massa entre 300 a $450{ }^{\circ} \mathrm{C}$ de aproximadamente $58,41 \%$. Embora não tenha apresentado diferença dos resultados quanto a divisão das amostras (A e B), a decomposição térmica das madeiras de Caesalpinia pyramidalis e Aspidosperma pyrifolium ocorre dentro da faixa de 300 a $450{ }^{\circ} \mathrm{C}$ e esse fato ocorre principalmente pela liberação do teor de matérias voláteis nesse intervalo de temperatura. Quanto a análise química imediata houve diferença estatística apenas para o teor de cinzas, sendo a Caesalpinia pyramidalis com maior teor $(4,98 \%)$. Portanto das características tecnológicas da madeira para energia as duas espécies apresentam-se com comportamento semelhantes e são potenciais para a geração de energia.

Palavras-Chave: termogravimetria, madeira para energia, análise química imediata.

\footnotetext{
${ }^{1}$ Discente do Programa de Pós-Graduação em Ciências Florestais, Universidade Federal do Rio Grande do Norte, cynthiapss@live.com

2 Discente do Programa de Pós-Graduação em Ciências Florestais, Universidade Federal do Rio Grande do Norte, sarahcostae@gmail.com

${ }^{3}$ Discente do Programa de Pós-Graduação em Ciências Florestais, Universidade Federal do Rio Grande do Norte, izabelle.rodriguesferreira@gmail.com

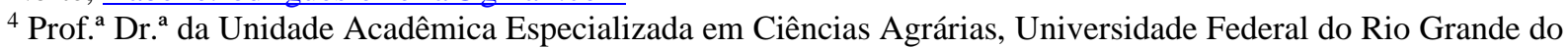
Norte, meire caico@yahoo.com.br

${ }^{5}$ Prof. Dr. da Universidade Federal de Viçosa, vresende@gmail.com
} 


\begin{abstract}
The process of converting wood into energy becomes complex from the inference about the heterogeneity of this material, which configures different proportions of the chemical composition of the wood among the different species. In order to understand the behavior of the wood in different temperatures, this work had as objective to verify the thermal decomposition of the wood of two forest species of the Caatinga, using the thermogravimetric method (TGA), and to evaluate the content of ash, fixed carbon and volatiles to indicate their potential for energy purposes. Three individuals of the species Caesalpinia pyramidalis and two individuals of the species Aspidosperma pyrifolium were collected, located at Fazenda Milhã / Poço da Pedra, RN. Discs with $15 \mathrm{~cm}$ of thickness were removed from each individual in the range of the diameter of the chest $(1.30 \mathrm{~m})$. The ASTM methodology E8721982 (ASTM, 2006) for volatile matter, ASTM E1755-2001 (ASTM, 2007) was adopted for the ash content and the fixed carbon was estimated by the difference of these two levels. The thermogravimetric analysis was performed using a temperature range of 25 to $750{ }^{\circ} \mathrm{C}$ under a $\mathrm{N}^{2}$ atmosphere. and heating rate of $10^{\circ} \mathrm{C}$. min-1 and two replicates were adopted for each species "A and B". In the statistical analysis, the Kolmogorov-Smirnov, Levene, F-tests of the analysis of variance were used and $95 \%$ probability was considered. For samples "A" the mass loss between 300 and $450{ }^{\circ} \mathrm{C}$ of approximately $53.65 \%$ was verified for samples $\mathrm{C} 4 \mathrm{~A}$ (Caesalpinia pyramidalis) and P2A (Aspidosperma pyrifolium) and $60 \%$ for samples $\mathrm{C} 2 \mathrm{~A}$ and $\mathrm{C} 3 \mathrm{~A}$ (Caesalpinia pyramidalis). For the "B" samples the mass loss between 300 and $450{ }^{\circ} \mathrm{C}$ of approximately $58.41 \%$ was verified. Although there was no difference in the results regarding the division of the samples (A and $\mathrm{B}$ ), the thermal decomposition of Caesalpinia pyramidalis and Aspidosperma pyrifolium wood occurs within the range of 300 to $450{ }^{\circ} \mathrm{C}$ and this fact occurs mainly by the release of the content of materials volatiles in this temperature range. As for the immediate chemical analysis, there was statistical difference only for ash content, with Caesalpinia pyramidalis having the highest content (4.98\%). Therefore of the technological characteristics of the wood for energy the two species present with similar behavior and are potential for the generation of energy.
\end{abstract}

Keywords: thermogravimetry, wood for energy, immediate chemical analysis.

\title{
INTRODUÇÃO
}

O interesse no uso de bioenergias está aumentando devido à necessidade de mitigar os efeitos das mudanças climáticas, redução de custos e pela oferta finita de combustíveis fósseis e o preço decrescente da madeira (DYMOND et al., 2010). Nesse contexto, a utilização da madeira como fonte energética oferece oportunidades para substituição do consumo de fontes não renováveis de energia por um recurso renovável.

O Brasil, por naturalmente apresentar uma maior biodiversidade, exibe potencial na geração de energia a partir da utilização da madeira, quando comparado a outros países. A demanda por lenha está presente em toda a região Nordeste nos diversos setores, entretanto, os industriais têm sua demanda mais expressiva. No estado do Rio Grande do Norte, o setor ceramista participa ativamente do crescimento econômico e tem alta dependência de lenha para alimentar os fornos responsáveis pela queima da madeira (CHAVES, 2016).

No entanto, o processo de conversão da madeira em energia a partir de altas temperaturas, como acontece nos fornos, provoca a decomposição térmica dos componentes 
presentes neste material. Além disso, cada componente apresenta uma característica específica de degradação e pode variar de espécie para espécie, podendo ser decomposto com maior ou menor facilidade, a depender da temperatura de carbonização e da espécie. Em razão da complexidade deste processo, é fundamental avaliar o perfil da decomposição térmica dos materiais utilizados pelas indústrias, considerando que a madeira é um material heterogêneo e cada espécie possui um comportamento quanto à velocidade de perda de massa durante o processo de carbonização (CARNEIRO et al., 2013).

Com esse método é possível determinar em qual temperatura o processo de degradação térmica se inicia e quando é mais acentuado, com base na massa da amostra, além de fornecer a quantidade de resíduo que é produzida em determinada temperatura, sendo, dessa maneira, ferramenta importante quando se deseja selecionar espécies para fins energéticos (CARNEIRO et al., 2013).

Respostas dessa natureza são importantes considerando a questão do uso sustentável da madeira da Caatinga, através da seleção de espécies que apresentem maior resistência térmica durante o processo de queima e, consequentemente, maior potencial no fornecimento de energia. Nesse contexto, este trabalho teve como objetivo analisar o perfil de decomposição da madeira de duas espécies da Caatinga (Caesalpinia pyramidalis e Aspidosperma pyrifolium) utilizando as técnicas de análise termogravimétrica.

\section{FUNDAMENTAÇÃO TEÓRICA}

A madeira é um material combustível e, quando aquecida, produz gases combustíveis que, quando expostos ao calor, queimam e produzem chama. Os gases quentes e as chamas, por sua vez, aquecem a porção de madeira ainda não atingida pelo calor e promovem a liberação de mais gases inflamáveis, gerando um processo em cadeia que alimenta a combustão. Tanto a madeira quanto o carvão oriundo da combustão da primeira são isolantes térmicos e retardam o fluxo de calor para o interior da seção, abrandando a velocidade da degradação térmica e retardando o avanço da frente de carbonização (FIGUEROA, 2009).

A obtenção de energia da madeira a partir do fornecimento de altas temperaturas promove a decomposição térmica dos seus componentes químicos, passando por um processo de carbonização sob atmosfera inerte e, ou, combustão sob atmosfera oxidante. No entanto, cada um desses componentes apresenta essa decomposição mais pronunciada em faixas de temperaturas distintas (CARNEIRO et al.,2013).

Objetivando observar e compreender a decomposição térmica da biomassa florestal, vários modelos que utilizam um limitado, mas sucessivo, número de reações paralelas tem 
sido desenvolvido para descrever este processo, incluindo modelos que lidam apenas com a transformação química de uma fase para a outra. Outros, porém, consideram a biomassa como uma mistura de seus principais constituintes (celulose, hemiceluloses e lignina) e empregam as reações para a conversão química destes usando um modelo para a contabilização das reações das principais macromoléculas, a fim de descrever a decomposição térmica de cada um deles (ZABANIOTOU et al., 2011).

As análises termogravimétricas (TGA) são as técnicas mais utilizadas para investigar e comparar os eventos térmicos durante a pirólise de pequenas amostras de combustível e medir as variações de massa durante o aquecimento. Esta técnica permite interpretar o comportamento da madeira durante a sua decomposição, determinar a estabilidade térmica do material, além de fornecer informações de quais faixas de temperatura ela é mais pronunciada (SANTOS et al., 2012).

Conforme apontado por Saddawi et al. (2010), a decomposição da biomassa florestal é um processo muito complexo em razão da ocorrência de grande número de reações e a natureza delas. A fase de transição da biomassa sólida para gasosa também aumenta a dificuldade e complica a interpretação das análises, sobretudo quando modelos detalhados que lidam com o fenômeno de transporte entre as fases são utilizados. A avaliação da função de transferência de calor e massa entre as fases e a sua importância em todo o processo podem afetar, significativamente, os resultados.

\section{METODOLOGIA}

A coleta da madeira para o estudo foi realizada na Fazenda Milhã/Poço da Pedra, localizada na região Agreste, microrregião de Serra Verde, entre os municípios de Jardim de Angicos e João Câmara, a $100 \mathrm{~km}$ da capital do estado do Rio Grande do Norte. A fazenda possui 1.132,78 ha de mata nativa de Caatinga sob manejo florestal sustentável.

Foram selecionados três indivíduos da espécie Caesalpinia pyramidalis e dois indivíduos da espécie Aspidosperma pyrifolium. De cada indivíduo, foram retirados discos de madeira com $15 \mathrm{~cm}$ de espessura localizados na altura do diâmetro do peito - DAP (1,30 m de altura). As amostras de madeira foram transformadas em serragem, com o auxílio de um moinho de laboratório tipo Wiley, de acordo com a norma 257 om-52 e posteriormente peneiradas, selecionando-se a fração que ficou retida na peneira $\mathrm{n}^{\circ} 24$ internacional, com malha de 60 mesh (ASTM, 1982).

Para a determinação da composição imediata, foi utilizada a metodologia descrita na norma ASTM E872-1982 (ASTM, 2006) para materiais voláteis, ASTM E1755-2001 (ASTM, 
2007) para teores de cinzas e o carbono fixo foi definido pelo cálculo da diferença:

$$
\% C F=100-(\% \mathrm{MV}+\% \mathrm{CZ})
$$

em que:

$\% \mathrm{CF}=$ porcentagem de carbono fixo;

$\% \mathrm{MV}=$ porcentagem de materiais voláteis;

$\% \mathrm{CZ}=$ porcentagem de cinzas.

A análise termogravimétrica foi realizada em um Analisador Termogravimétrico TGA-1000, da 39 marca Navas Instruments. As análises foram feitas em um cadinho de platina com uma massa aproximada de $1 \mathrm{~g}$ de amostra em uma faixa de temperatura de $25 \mathrm{a}$ $750{ }^{\circ} \mathrm{C}$ sob atmosfera de $\mathrm{N}^{2}$ (fluxo de $100 \mathrm{~mL} \cdot \mathrm{min}^{-1}$ ) e razão de aquecimento de $10{ }^{\circ} \mathrm{C}$. min-1. Para a análise termogravimétrica as amostras de cada espécie foram divididas em repetições A e B.

Os dados foram submetidos ao teste de Kolmogorov-Smirnov para verificação da normalidade. A homocedasticidade das variâncias verificada pelo teste de Levene. Para as variáveis independentes do tipo quantitativas, conduziram-se as correlações de Pearson e teste F da análise de variância da regressão (ANAVA). Todos os testes foram realizados $95 \%$ de probabilidade com o auxílio do software R Core Team.

\section{RESULTADOS E DISCUSSÃO}

Na Tabela 1 estão apresentados os dados do teor de cinzas, matérias voláteis e carbono fixo da madeira das duas espécies florestais estudadas.

Tabela 1 - Teor de cinzas, matérias voláteis e carbono fixo das espécies Caesalpinia pyramidalis (Catingueira) e Aspidosperma pyrifolium (Pereiro). Fonte: própria.

\begin{tabular}{cccc}
\hline Espécies & $\begin{array}{c}\text { Teor de cinzas } \\
(\%)\end{array}$ & $\begin{array}{c}\text { Matérias voláteis } \\
(\%)\end{array}$ & $\begin{array}{c}\text { Carbono fixo } \\
(\%)\end{array}$ \\
\hline Caesalpinia pyramidalis & $4,98 \mathrm{a}$ & $78,76 \mathrm{a}$ & $16,25 \mathrm{a}$ \\
\hline Aspidosperma pyrifolium & $0,23 \mathrm{~b}$ & $78,75 \mathrm{a}$ & $21,02 \mathrm{a}$ \\
\hline * Médias seguidas da mesma letra entre as espécies, para uma mesma variável, não diferem entre si, a $5 \%$ de \\
significância, pelo teste Tukey.
\end{tabular}

Analisando-se a Tabela 1, observa-se que com relação à composição química imediata das espécies estudadas, o teor de cinzas foi o único parâmetro que apresentou diferenças estatísticas entre as espécies. A Caesalpinia pyramidalis apresentou maior valor médio percentual de cinzas do que a Aspidosperma pyrifolium.

Ao considerar o uso da madeira para destinação energética, certamente, um material com menor teor de cinzas é mais indicado, uma vez que esse parâmetro se refere à quantidade 
de matéria inorgânica presente na composição da madeira, o que corrobora com o resultado para espécie Aspidosperma pyrifolium, em que provavelmente a redução do rendimento energético do sistema é menor. Em relação aos teores de cinzas, estes afetam negativamente o poder calorífico da biomassa e aumentam a frequência de limpeza dos cinzeiros das fornalhas devido ao aumento na produção de cinzas, bem como acarretam corrosão e incrustações nos metais utilizados na construção dos sistemas de combustão (VITAL et al., 2013).

Os valores médios determinados para matérias voláteis e carbono fixo são próximos aos encontrados por Brito; Barrichelo (1982), que mencionaram que os teores de voláteis da madeira se encontram entre $75 \%$ a $85 \%$ e o carbono fixo entre $15 \%$ a $25 \%$. De acordo com Souza e Vale (2016) a reação da biomassa à queima está relacionada com o teor de materiais voláteis, ou seja, a facilidade de ignição da biomassa devido à volatilização de substâncias do material durante o aquecimento. Para Cortez; Lora (2008) o percentual de material volátil é importante principalmente para a ignição e nas etapas iniciais da combustão da biomassa.

Nas Figuras 1 e 2 é possível observar um pico endotérmico na faixa de 100 a $150{ }^{\circ} \mathrm{C}$ situado no início da DTG, que está relacionado ao teor de umidade da amostra, a liberação de vapor de água da madeira. Em termos energéticos, a reação exotérmica inicia entre a faixa de $350{ }^{\circ} \mathrm{C}$, antes disso há o consumo de energia para a liberação do vapor de água na madeira. Sendo assim, o consumo de energia fornecido para essa reação endotérmica dependerá da quantidade de água a ser liberada na madeira.

Figura 1 - Curvas termogravimétricas TG e DTG da amostra A das espécies Caesalpinia pyramidalis e Aspidosperma pyrifolium. Fonte: própria.
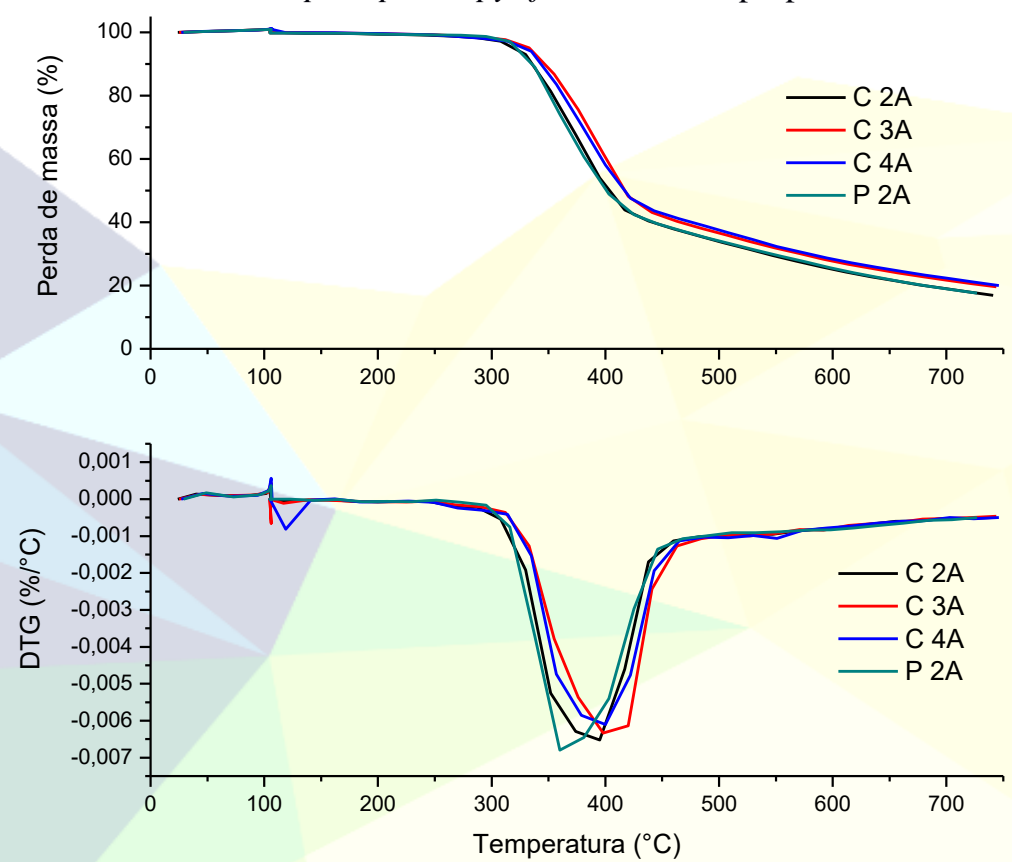

* C: Caesalpinia pyramidalis (Catingueira); P: Aspidosperma pyrifolium (Pereiro). 
Figura 2 - Curvas termogravimétricas TG e DTG da amostra B das espécies Caesalpinia pyramidalis e Aspidosperma pyrifolium. Fonte: própria.
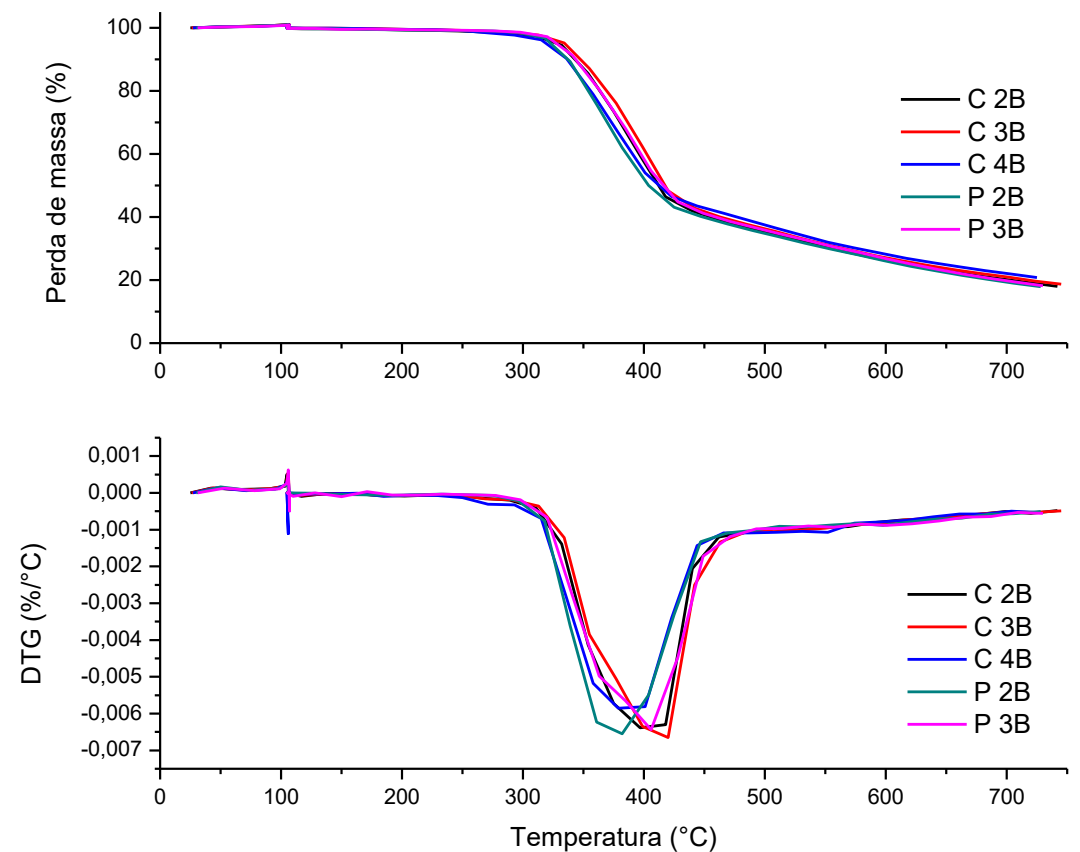

* C: Caesalpinia pyramidalis (Catingueira); P: Aspidosperma pyrifolium (Pereiro).

De acordo com a Figura 1, observa-se que para as amostras "A" das espécies Caesalpinia pyramidalis e Aspidosperma pyrifolium há uma perda de massa entre 300 $450{ }^{\circ} \mathrm{C}$ de aproximadamente $53,65 \%$ para as amostras C4A e P2A e de $60 \%$ para as amostras $\mathrm{C} 2 \mathrm{~A}$ e C3A. A máxima perda de massa na faixa de $400{ }^{\circ} \mathrm{C}$ pode estar relacionada à maior degradação da celulose e liberação das matérias voláteis (CARNEIRO et al., 2013).

De acordo com a Figura 2, observa-se que para as amostras "B" das espécies Caesalpinia pyramidalis e Aspidosperma pyrifolium há perda de massa entre $300-450{ }^{\circ} \mathrm{C}$ de aproximadamente $58,41 \%$. Da mesma forma que as amostras "A", a perda de massa nessa faixa está diretamente relacionada aos teores de materiais voláteis presentes na composição química da madeira. Nesse sentido, nota-se que não há diferença significativa da degradação térmica entre indivíduos de uma mesma espécie e até entre as duas espécies. $\mathrm{O}$ comportamento da perda de massa, bem como, da DTG ocorre de forma semelhante para ambas as espécies.

\section{CONCLUSÕES}

A decomposição térmica das madeiras de Caesalpinia pyramidalis e Aspidosperma pyrifolium ocorre dentro da faixa de 300 a $450{ }^{\circ} \mathrm{C}$ e esse fato ocorre principalmente pela liberação do teor de matérias voláteis nesse intervalo de temperatura. Houve diferença estatística quanto ao teor de cinzas das espécies, sendo a Caesalpinia pyramidalis com maior 
teor $(4,98 \%)$. Apesar de o teor de matérias voláteis e carbono fixo das espécies estudadas serem estatisticamente iguais, o percentual de carbono fixo é maior para a espécie Aspidosperma pyrifolium (Pereiro) o que possibilita inferir sobre o seu maior rendimento energético durante a decomposição térmica.

\section{REFERÊNCIAS}

- ASTM - AMERICAN SOCIETY FOR TESTING AND MATERIALS. Standard E872, 1982 (2006), Standard Test Method for Volatile Matter in the Analysis of Particulate Wood Fuels. ASTM International, West Conshohocken, PA, 2006.

- ASTM - AMERICAN SOCIETY FOR TESTING AND MATERIALS. Standard E1755, 2001 (2007), Standard Test Method for Ash in Biomass. ASTM International, West Conshohocken, PA, 2007.

BRITO, J. O. BARRICHELO, L. E. G. MURAMOTO, M. C. HILTON, T. Z. C. Estimativa da densidade a granel do carvão vegetal a partir de sua densidade aparente. Instituto de Pesquisas e Estudos Florestais. Circular Técnica n ${ }^{\circ}$ 150. Outubro/1982.

CARNEIRO, A. C. O. et al. Estudo da decomposição térmica da madeira de oito espécies da Região do Seridó, Rio Grande do Norte. Revista Árvore, v. 37, n. 6, 2013.

CHAVES, A. G. da C. Diagnóstico da exploração de lenha em planos de manejo sustentável na caatinga do Rio Grande do Norte. 2016. 47f. Dissertação (Mestrado em Ciências Florestais) - Universidade Federal do Rio Grande do Norte, Universidade Federal do Rio Grande do Norte, 2016.

CORTEZ, L. A. B. C.; LORA, E. E. S.; GÓMEZ, E. O. Biomassa para energia. Editora da Unicamp - Campinas, SP, 2008.

DYMOND, C. C. et al. Future quantities and spatial distribution of harvesting residue and dead wood from natural disturbances in Canada. Forest Ecology and Management, v. 260, n. 2, p. 181-192, 2010.

FIGUEROA, M. J. M.; MORAES, P. D. Comportamento da madeira a temperaturas elevadas. CEP, v. 88040, p. 970, 2009.

SADDAWI, A. et al. Kinetics of the thermal decomposition of biomass. Energy Fuels, v. 24, p.1274-1282, 2010.

SANTOS, R. C. et al. Análise Termogravimétrica em clones de eucalipto como subsídio para a produção de carvão vegetal. Cerne, v. 18, n. 1, p. 143-151, 2012.

SOUZA, F.; VALE, A. T. Densidade energética de briquetes de biomassa lignocelulósica e sua relação com os parâmetros de briquetagem. Pesq. flor. bras., v. 36, n. 88, p. 405-413, 2016.

VITAL, B. R.; CARNEIRO, A. C. O.; PEREIRA, B. L. C. Qualidade da madeira para fins 
energéticos. In: Santos, F.; Colodette, J.; Queiroz, J. H. (Org.). Bioenergia \& Biorrefinaria: Cana-de-Açúcar \& Espécies Florestais. 1ed.Visconde do Rio Branco: Suprema, 2013, v. 1, p. 321-354.

ZABANIOTOU, A. et al. Thermal degradation studies and kinetic modeling of cardoon (Cynara cardunculus) pyrolysis using thermogravimetric analysis. Bioresource Technology, v. 102, p. 6230-6238, 2011. 